\title{
Ingested foreign bodies associated with orthodontic treatment: report of three cases and review of ingestion/aspiration incident management
}

\author{
T. M. Milton, ' S. D. Hearing, ${ }^{2}$ and A. J. Ireland, ${ }^{3}$
}

\section{Foreign body ingestion/aspiration episodes are potential complications in all branches of dentistry. The handling of small orthodontic components requires particular care, especially where the patient is supine or semi-recumbent. Three cases of foreign body ingestion are presented, involving patients undergoing orthodontic treatment. Once the foreign bodies had been located, all instances were treated using a combination of serial radiography and 'watchful waiting'. All patients remained asymptomatic during this period, although none of the foreign bodies were retrieved. No active intervention was deemed necessary, and the patients were able to resume their orthodontic treatment. The potential complications of ingestion/aspiration episodes are discussed and a management regime suggested.}

$\mathrm{O}$ rthodontic components are mostly small, and in combination with saliva, handling can sometimes be difficult. With a patient who is supine or semi-recumbent, there is the risk of a dropped object falling into the oropharynx, and being swallowed or inhaled. The patient may also be prone if components become detached or fractured during service. Depending on the size, shape and flexibility of the object, some occurrences present minimal danger, whilst others have the potential to be lethal.

${ }^{*}$ Senior House Officer, Department of

Oral/Maxillofacial Surgery and Orthodontics, Royal United Hospital, Combe Park, Bath BA1 3NG;

${ }^{2}$ Lecturer in Gastroenterology, University Division of

Medicine, Bristol Royal Infirmary, Bristol BS2 8HW;

${ }^{3}$ Consultant in Orthodontics, Department of

Oral/Maxillofacial Surgery and Orthodontics, Royal

United Hospital, Combe Park, Bath BA1 3NG

${ }^{*}$ Correspondence to: $\mathrm{Mr}$ T M Milton, c/o Department of Oral/Maxillofacial Surgery, Southmead Hospital,

Bristol BS10 5NB

email:milton_t@southmead.swest.nhs.uk

REFEREED PAPER

Received 06.07.00; Accepted 20.11.00

(C) British Dental Journal 2001; 190: 592-596

\section{In brief}

- Foreign body ingestion/aspiration episodes have the potential to result in acute medical and life-threatening emergencies. Prevention of such incidents is therefore the best approach, via the mandatory use of procedures, aimed at securing potentially loose dental objects.

- The GDP must be able to recognise signs and symptoms of air obstruction, if a dental object is lost into the oropharynx. If retrieval is not possible, emergency life support measures must be instigated, whilst waiting for help to arrive.

- If the airway is not compromised, the patient must be escorted to the hospital for clinical and radiographical examination, to identify the location of the object. Treatment thereafter examination. precautions during all dental will depend on the findings of the
Prevention is obviously the best approach, but proper management of such an incident when it occurs is crucial to the health and safety of the patient. The objective of this paper is to present three such cases, to draw attention to the potentially serious consequences, and to suggest a protocol for management.

\section{Report of Cases}

Case 1

A 14-year-old girl had previously been treated at the Orthodontic Department of the Royal United Hospital, Bath for correction of a Class II incisor relationship. Because of a small degree of lower incisor relapse, a new lower fixed appliance was fitted, to align the teeth. Bonds were placed from LR6(46) to LL6(36). On review 2 weeks later, it was noted that the bond on LR6(46) had been lost. Following referral to the Radiology Department, posterior-anterior abdominal films revealed the lost bracket to be located within the large bowel (Fig. 1). The patient and parents were made aware of the situation and advice was given regarding examination of stools. A further review appointment was arranged for 2 weeks time. The patient remained asymptomatic during this period, but the bracket was not identified in the stools. LR6(46) was not rebonded, and the treatment was completed using attachments from LR5(45) to LL6(36). Treatment progressed through the use of 0.012 " nickel titanium, up to 0.018 " x 0.025 " stainless steel arch wires. After 13 months the patient was debonded, and a lower fixed retainer fitted.

\section{Case 2}

A 13-year-old girl was referred to the Orthodontic Department at the Royal United 


\section{PRACTICE $\underline{\text { medical matters }}$}

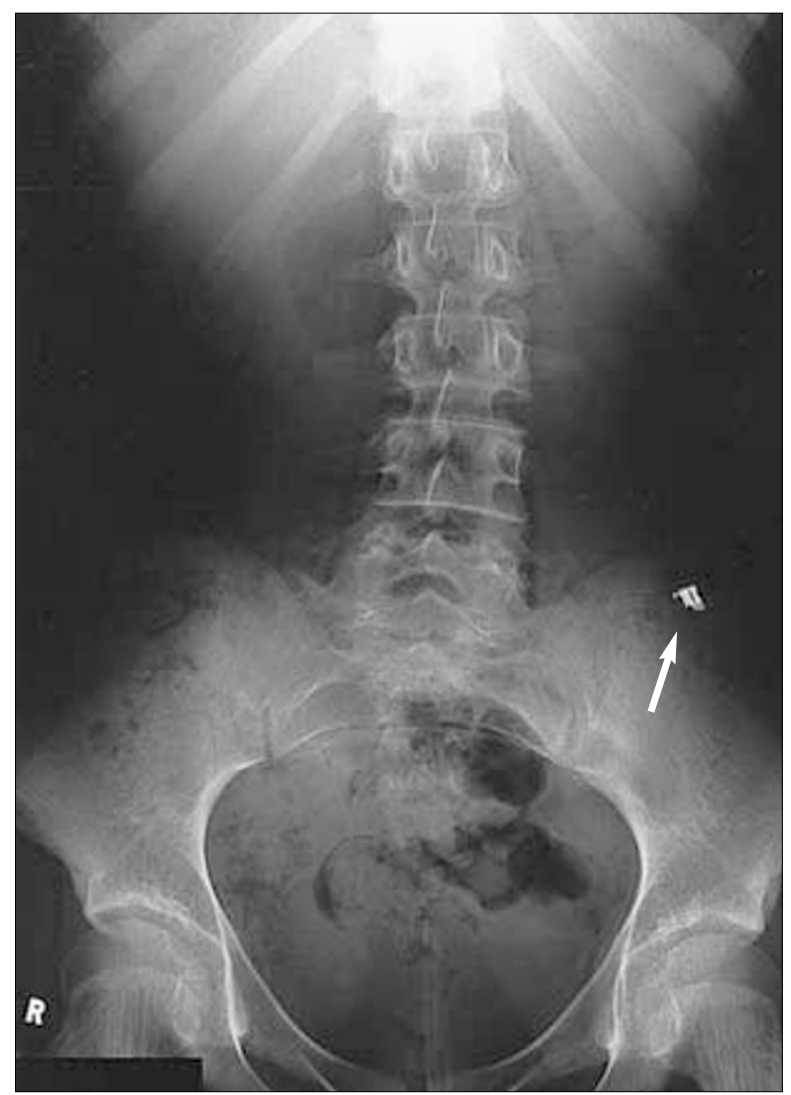

Fig. I Posterior-anterior abdominal radiograph showing bonded tube in descending colon (Case I)

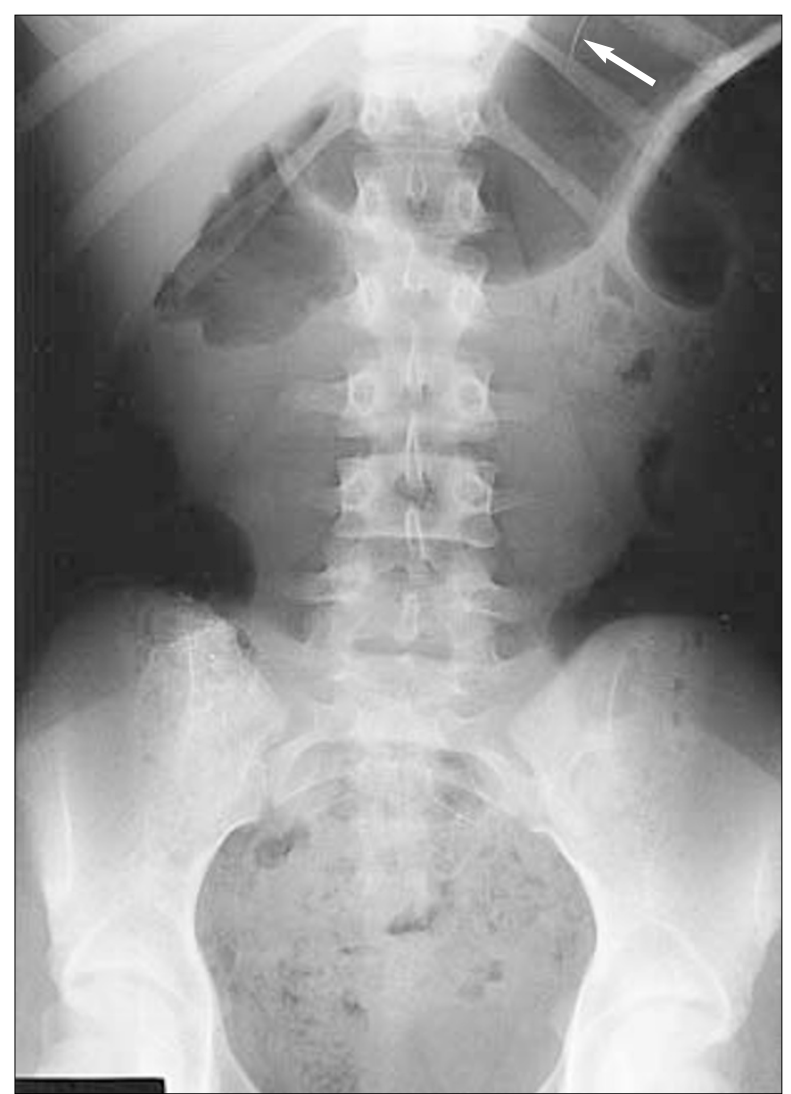

Fig. 2 Posterior-anterior abdominal radiograph showing large fragment of 0.016" archwire in stomach (Case 2)
Hospital for treatment of a Class I incisor relationship with crowding. The treatment plan was extraction of UR5(15),UL5(25) and LR5(45) and LL5(35), and upper and lower fixed appliance therapy. Arch wire progression following bond placement was 0.012 " nickel titanium followed 2 months later by placement of a 0.016 " nickel titanium arch wire. Two months after placement of the second wire the patient

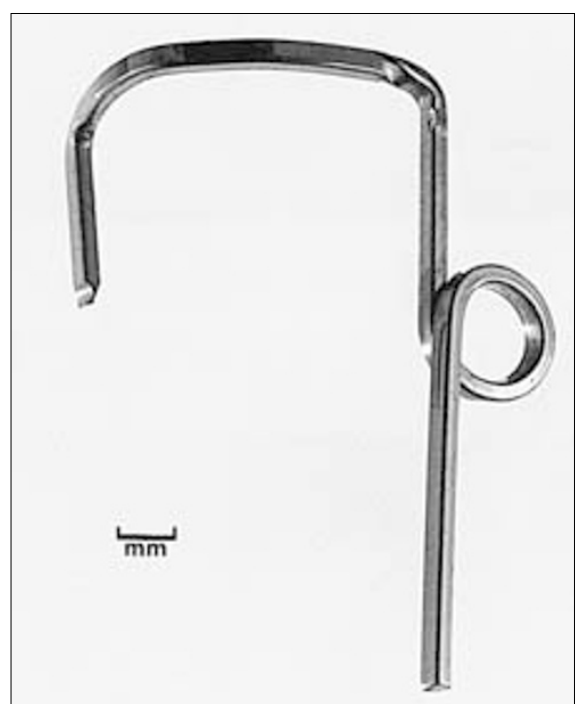

Fig. 30.017 " $\times 0.025$ " stainless steel sectional wire of the type swallowed by the patient in Case 3 returned for an emergency appointment. She stated that a portion of the lower arch wire had become detached during breakfast that morning. The patient was referred to the Radiology Department at the Royal United Hospital for posterior-anterior chest and abdominal films. A segment of wire approximately $20 \mathrm{~mm}$ long was located in the stomach (Fig. 2). The patient and parents were informed, and a review appointment with repeat films was organized for 2 days time. The patient remained asymptomatic during this period, and the two repeat posterior-anterior films revealed that the wire segment was no longer present. The patient's orthodontic treatment is still ongoing and has otherwise been uneventful.

\section{Case 3}

A 15-year-old boy was referred to the Royal United Hospital following earlier treatment of his Class II/I incisor relationship. The initial treatment had consisted of upper and lower fixed appliance therapy, and the loss of UR4(14),UL4(24) and LR5(45),LL5(35). Since the completion of this basic treatment, LR7(47) had erupted, but was mesioangularly impacted against the distal of LR6(46). The decision was made to upright this tooth using a bonded molar tube on LR7(47) and a 0.017" x 0.025" stainless steel sectional archwire, passing from the tube onto the occlusal surface of LR6(46)(Fig. 3). This sectional wire was held in place with a polyurethane module passing around the wire and hook on the molar tube. Eight days into treatment, the patient returned having lost a sectional wire, and with no idea as to where it had gone. A posterior/anterior film of the abdomen (Fig. 4), showed the wire, measuring approximately $20 \mathrm{~mm}$ in length, to be within the patient's stomach. The patient and parents were informed and a review appointment arranged for the following day. A repeat film taken then showed the wire to be within the large intestine (Fig. 5); the patient remained asymptomatic. A further appointment was organized for the following week, when another posterior/ anterior abdominal film was taken. This showed no sign of the wire. The patient continued to remain well, and a new sectional wire was fitted. The LR7(47) continued to erupt into a good position.

\section{Discussion}

Foreign body ingestion/aspiration episodes in any branch of dentistry have the potential to result in acute medical and life-threatening emergencies. ${ }^{1,2}$ Prevention of ingestion/ aspiration incidents is therefore the best approach, via the mandatory use of precautions during all dental procedures. ${ }^{3}$ In the 


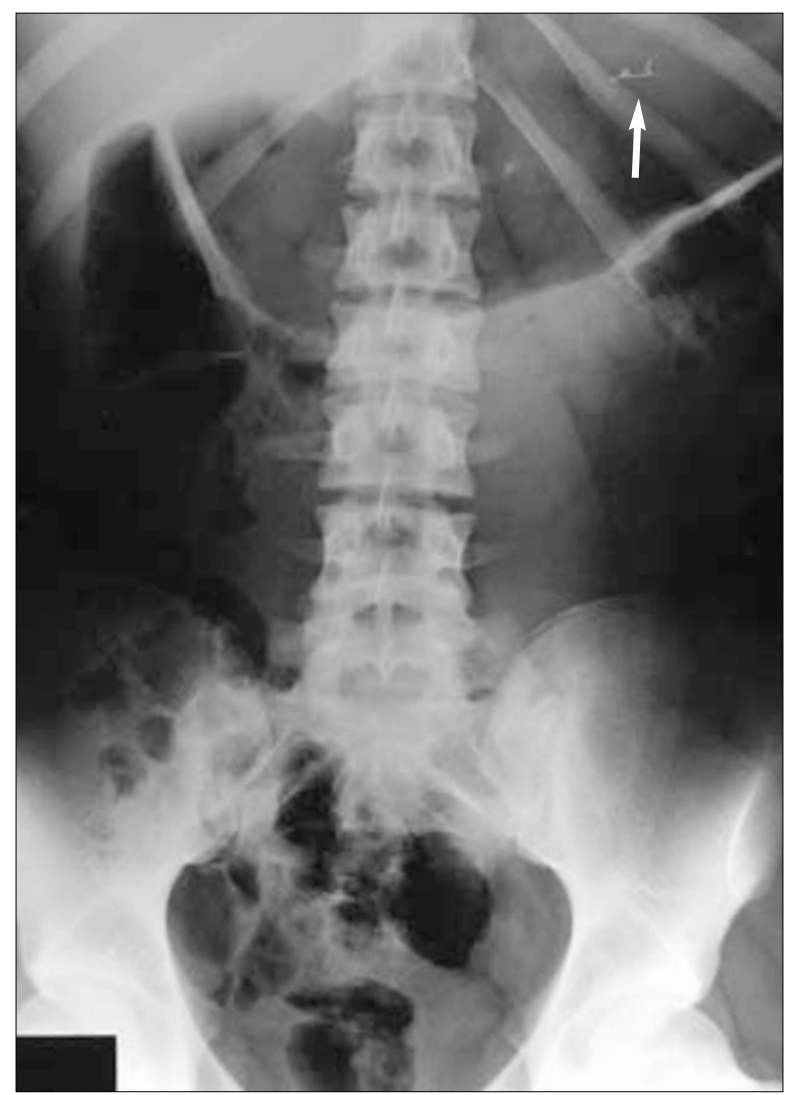

Fig. 4 Posterior-anterior abdominal radiograph showing sectional wire in stomach (Case 3)

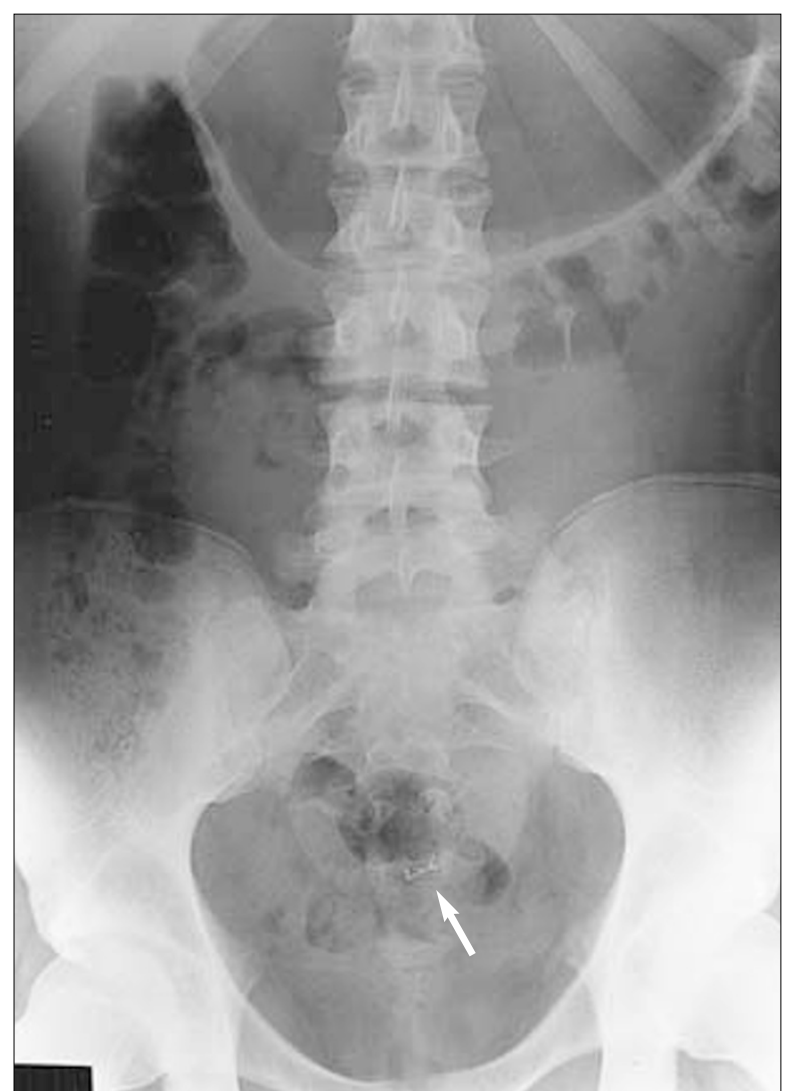

Fig. 5 Posterior-anterior abdominal radiograph showing sectional wire in large intestine (Case 3) orthodontic clinic, all arch wires should be cinched down distal to a bonded attachment, and secured before intra-oral cutting. The secure bonding of brackets to teeth must be confirmed, and components such as expansion keys adapted appropriately, to allow attachment of dental floss by the patient. 4,5 With the use of the latter, parents should be made aware of the possibility of swallowing or aspirating the key, and should be advised regarding emergency management of the patient in such an event.

Acrylic components of orthodontic appliances are likely to be radiolucent. As radiographs are used to locate foreign bodies (as in the cases above) this will lead to difficulties in their localisation, especially if they become separate from an associated radio-opaque framework. This problem can be overcome by incorporating radioopaque material into the acrylic. ${ }^{6}$

If a dental component/foreign body is lost into the oropharynx, the patient should be placed in a reclined position, and encouraged to cough vigorously. The immediate priority is to ensure a clear airway. If the latter is compromised, this will quickly give rise to recognisable symptoms, such as choking, inspiratory stridor, and forced breathing using the accessory musculature. If further vigorous coughing fails to bring any improvement, the Heimlich manoeuvre should be performed, to try and relieve the laryngeal obstruction. ${ }^{7}$ This procedure needs to be very rapidly successful; otherwise emergency help must be summoned immediately, for transfer of the patient to the nearest hospital emergency unit. Whilst awaiting this help, the practitioner and her/his team must instigate emergency life support measures, including airway provision via a cricothyroidotomy, if appropriate. ${ }^{8}$

If the patient's airway is not compromised, this situation should be maintained and monitored as a search of the oral cavity and local area is carried out. Retrieval, identification, and confirmation that the object is intact should be followed immediately by reassurance for the patient. If the object is not retrieved, however, the patient should be reassured, but informed about the complication and transferred to hospital, so that appropriate radiographic and clinical examination can be carried out. This should consist of chest and abdominal radiographs, which should enable the attending physician to determine the location of the object.

In the case of swallowed foreign bodies, the complications of intestinal obstruction, perforation with subsequent abscess formation, haemorrhage, or fistula and failure of objects to progress, may occur. ${ }^{9}$ The majority of foreign bodies that cause obstruction lodge in the upper oesophagus. ${ }^{10}$ This can lead to oesophageal perforation with secondary mediastinitis and oesophageal obstruction with the risk of aspiration. Thus, swallowed foreign bodies retained in the oesophagus should be urgently removed using fibre-optic endoscopy. Once a foreign body has reached the stomach however, there is a greater than $90 \%$ chance of the object being passed from the gastrointestinal tract without problems, usually over a 7-10 day period. ${ }^{11}$ The risk of a foreign body causing perforation or obstruction is related to the shape and size of the object. Sharp pointed objects are associated with a higher risk of perforation. In addition, objects longer than $5 \mathrm{~cm}$ are unlikely to pass the duodenum. In both cases, early endoscopic removal should be undertaken. ${ }^{12}$ Once an object has left the stomach, most will pass through the small bowel. The most common subsequent site of perforation or obstruction is the ileocaecal valve. ${ }^{9,13}$ The risk of obstruction is increased in patients with abnormal intestinal anatomy such as strictures. Removal can be attempted colonoscopically.

The management of patients who have swallowed foreign bodies relies on regular assessment and serial radiography. The patient should be evaluated for symptoms of intestinal perforation or obstruction, such as pain or vomiting, and examined for signs 


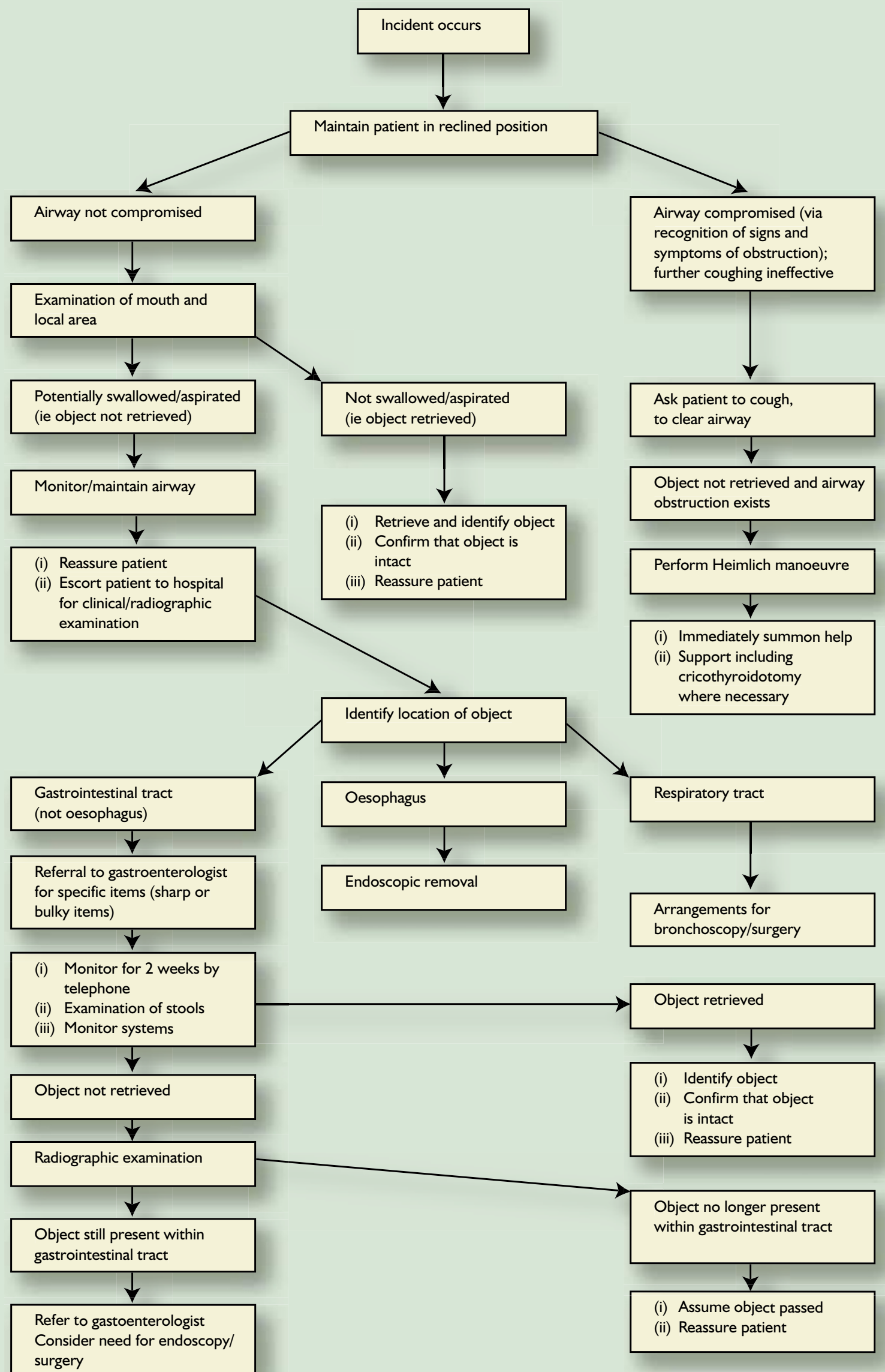

Fig. 6 Flow chart for management of ingested/aspirated dental objects 
such as tenderness or guarding. Provided that the patient is asymptomatic, an expectant policy can be adopted, and the stools examined regularly for the presence of the foreign body. There is no evidence that laxatives are helpful and they may increase the risk of perforation. ${ }^{10}$ Radiographs allow the clinician to confirm the presence of a foreign body, and to assess its size, shape and position, and to look for indications of intestinal perforation, such as pneumoperitoneum. In addition, radiographs can be used to monitor onward progress, and confirm passage of the foreign body. If the patient develops signs of perforation or the object remains lodged after 2 weeks, surgical intervention will usually be required.

In contrast to the swallowed foreign body, an aspirated foreign object can constitute a true medical emergency. As discussed, any ensuing laryngospasm and respiratory embarrassment are potentially life threatening, and immediate measures, including cricothyroidotomy, may be required to establish and maintain a patent airway. Even if the airway does not appear to be in immediate jeopardy, possibly early complications of foreign body aspiration may occur including acute dyspnoea, laryngeal oedema, perforation and pneumothorax. Long-term complications such as lung abscess and pneumonia may also develop. ${ }^{1,13}$ Thus, once a foreign body has been localised to the respiratory tract using appropriate radiography, retrieval should be carried out as soon as possible. ${ }^{14}$ Bronchoscopy is the method of choice, using a flexible fibre-optic bronchoscope with an appropriate attachment to facilitate foreign body retrieval. ${ }^{13,14}$ Fluoroscopic guidance for the removal may be necessary in places where the foreign body is lodged in very small bronchi towards the lung periphery. However, mucosal inflammation and oedema around the object may hinder manipulation of the bronchoscope; if retrieval fails, surgery (usually in the form of thorachotomy) becomes necessary. A suggested management regime for ingested/aspirated foreign bodies is outlined in Figure 6.

\section{Conclusion}

In addition to implementing preventative measures, practitioners must be aware of the correct management of patients who are suspected of having ingested/aspirated a foreign body. If necessary, this includes the immediate provision of potentially life saving treatment at the chairside. Once the airway has been secured, the patient must be escorted to hospital and referred for clinical and radiographic examination by the appropriate specialist. Treatment thereafter will follow on from the diagnosis as to the location of the foreign body.

The authors gratefully acknowledge the assistance of Mrs Julie Day at the Oral/Maxillofacial Department, Royal United Hospital, Bath, for her assistance in the preparation of this paper.

1 Fields R T, Schows S R. Aspiration and ingestion of foreign bodies in oral and maxillofacial surgery; a review of the literature, and report of five cases. Am J Oral Maxillofacial Surg 1998; 56: 1091-1098.

2 Cameron S M, Whitlock W L, Tabor M S. Foreign body aspiration in dentistry: a review. J Am Dent Assoc 1996; 127: 1224-1229.

3 Zitzmann N V, Elsasser S, Fried R, Marinello C P. Foreign body ingestion and aspiration. Oral Surg, Oral Med, Oral Pathol 1999; 6: 657-660.

4 Nazif M M, Ready M A. Accidental swallowing of orthodontic expansion appliance keys; report of two cases. ASDC J Dent Child 1983; 3 : 126-127.

5 Barkmeier W W, Abrams H, Barringer P S. Accidental swallowing of a pin wrench; report of a case. Ann Dent 1978; 37: 92-94.

6 Hinkle F G. Ingested retainer: a case report. Am JOrthod Dentofacial Orthop 1987; 1: 46-49.

7 Heimlich H J. The Heimlich manoeuvre: prevention of death from choking on foreign bodies. J Occup Med 1977; 19: 208-210.

8 Colquhoun M C, Handley A J, Evans T R. ABC of resuscitation. Third Edition. London: BMJ Publishing Group, 1995.

9 Maleki M, Evans W E. Foreign body perforation of the intestinal tract. Report of 12 cases, and a review of the literature. Arch Surg 1970; 101: 475-477.

10 Henderson C T, Engel J, Schlesinger P. Foreign body ingestion; review and suggested guidelines for management. Endoscopy 1987; 19: 68-71.

11 Webb W A. Management of foreign bodies of the upper gastrointestinal tract. Gastroenterol 1988; 94: 204-216.

12 Soergel K H, Hogan W J. Therapeutic endoscopy. Hosp Pract 1983; 18: 81-92.

13 Ziter F M. Intestinal perforation in adults due to ingested opaque foreign bodies. Am J Gastroenterol 1976; 66: 382-85

14 Deskin R, Young G, Hoffman R. Management of paediatric aspirated foreign bodies. Laryngoscope 1997; 4: 540-543.

15 Bergermann M, Donald P J, Wengen D F. Screwdriver aspiration: a complication of dental implant placement. Int J Oral Maxillofac Surg 1992; 21: 339-41. 